\title{
Editorial
}

\section{Statistical Estimation of Portfolios for Dependent Financial Returns}

\section{Masanobu Taniguchi, ${ }^{1}$ Cathy W. S. Chen, ${ }^{2}$ Junichi Hirukawa, $^{3}$ Hiroshi Shiraishi, ${ }^{4}$ Kenichiro Tamaki, ${ }^{1}$ and David Veredas ${ }^{5}$}

\author{
${ }^{1}$ Department of Applied Mathematics, Waseda University, Tokyo 169-8555, Japan \\ ${ }^{2}$ Department of Statistics/Graduate Institute of Statistics $\mathcal{E}$ Actuarial Science, \\ Feng Chia University Taichung 407, Taiwan \\ ${ }^{3}$ Department of Mathematics, Faculty of Science, Niigata University, Niigata 950-2181, Japan \\ ${ }^{4}$ The Jikei University School of Medicine, Tokyo 105-8461, Japan \\ ${ }^{5}$ ECARES-Solvay Brussels School of Economics and Management, Universite Libre de Bruxelles, \\ 1050 Brussels, Belgium
}

Correspondence should be addressed to Masanobu Taniguchi, taniguchi@waseda.jp

Received 22 August 2012; Accepted 22 August 2012

Copyright (C) 2012 Masanobu Taniguchi et al. This is an open access article distributed under the Creative Commons Attribution License, which permits unrestricted use, distribution, and reproduction in any medium, provided the original work is properly cited.

The field of financial engineering has developed as a huge integration of economics, mathematics, probability theory, statistics, time series analysis, operation research, and so forth, over the last decade. The construction of portfolios for financial assets is one of the most important issues in financial engineering. It is empirically observed that financial returns are non-Gaussian and dependent, and it is shown that the classical mean-variance portfolio estimator is not statistically optimal. Knowledge and understanding of these have led to the development of general time series modeling for financial returns, sophisticated optimal estimation theory, robust estimation methods, empirical likelihood for time series, nonstational time series analysis, prediction of time series, and various numerical approaches for portfolios.

As the contents, this special volume includes the following topics in financial time series analysis and financial engineering.

The paper titled "Large deviation results for discriminant statistics of Gaussian locally stationary processes" by J. Hirukawa discusses the large deviation principle of discriminant statistics for Gaussian locally stationary processes. The large deviation theorems for quadratic forms and the log-likelihood ratio for a Gaussian locally stationary process with a mean function are proved. Their asymptotics are described by the large deviation rate functions. Next, the situation where processes are misspecified to be stationary is considered. In these 
misspecified cases, the log-likelihood ratio discriminant statistics are formally constructed and the large deviation theorems of them are derived. Since they are mathematically complicated, they are evaluated and illustrated by numerical examples. We see that the misspecification of the process to be stationary seriously affects these discrimination.

The paper by T. Amano is titled "Asymptotic optimality of estimating function estimator for CHARN model." CHARN model is a famous and important model in the finance, which includes many financial time series models and can be used to model the return processes of assets. One of the most fundamental estimators for financial time series models is the conditional least squares CL estimator. However, recently, it was shown that the optimal estimating function estimator ( $G$ estimator) is better than CL estimator for some time series models in the sense of efficiency. This paper examines efficiencies of CL and G estimators for CHARN model and derives the condition that $\mathrm{G}$ estimator is asymptotically optimal.

The next paper titled "Optimal portfolio estimation for dependent financial returns with generalized empirical likelihood" by H. Ogata proposes to use the method of generalized empirical likelihood to find the optimal portfolio weights. The log-returns of assets are modeled by multivariate stationary processes rather than i.i.d. sequences. The variance of the portfolio is written by the spectral density matrix, and we seek the portfolio weights minimizing it. The illustration of this method to the real market index data is also given.

The paper titled "Statistically efficient construction of $\alpha$-risk-minimizing portfolio" by Hiroyuki Taniai and Takayuki Shiohama proposes a semiparametrically efficient estimator for $\alpha$-risk-minimizing portfolio weights. The optimal portfolio whose $\alpha$-risk being minimized is formulated in a linear quantile regression problem. The authors apply the rank-based semiparametric method, using the signs and ranks of residual, to provide the efficient construction of the optimal portfolios. This efficiency gain is verified by Monte Carlo simulations and Empirical applications.

The paper titled "Estimation for non-Gaussian locally stationary processes with empirical likelihood method" by H. Ogata considered an estimation problem for non-Gaussian locally stationary processes by empirical likelihood. The parameter of interest is specified by the time-varying spectral moment condition, and it can express various important indices for locally stationary processes such as an autocorrelation function. The asymptotic distribution of maximum empirical likelihood estimator and empirical likelihood ratio test statistic are given based on the central limit theorem for locally stationary processes.

The paper "A simulation approach to statistical estimation of multiperiod optimal portfolios" by $\mathrm{H}$. Shiraishi discusses a simulation-based method for solving discrete-time multiperiod portfolio choice problems under AR(1) return process. Based on the AR bootstrap, first, simulation sample paths of the random returns are generated. Then, for each sample path and each investment time, an optimal portfolio estimator, which optimizes a constant relative risk aversion (CRRA) utility function, is obtained.

The paper by J. Hirukawa entitled "On the causality between multiple locally stationary processes" is concerned with the concepts of dependence and causality which can describe the relations between multivariate time series. These concepts also appear to be useful when one is describing the properties of an engineering or econometric model. Although the measures of dependence and causality under stationary assumption are well established, empirical studies show that these measures are not constant in time. In this paper, the generalized measures of linear dependence and causality to multiple locally stationary processes are proposed. The measures of linear dependence, linear causality from one series to the other, and instantaneous linear feedback, at each time and each frequency, are given. 
The paper titled "Optimal portfolios with end-of-period target" by H. Shiraishi et al. studies the estimation of optimal portfolios for a Reserve Fund with an end-of-period target, when the returns of the assets constituting the Reserve Fund portfolio follow two specifications. They focus the case when assets are split into short memory bonds and long memory equity or when returns of the distribution are heavy-tailed stable.

The next paper is by J. Hirukawa and M. Sadakata entitled "Least squares estimators for unit root processes with locally stationary disturbance." It contains a discussion of various properties of the least squares estimators for unit root processes with locally stationary innovation processes. Since locally stationary process is not a stationary process, these models include different two types of nonstationarity, namely, unit root and locally stationarity. The locally stationary innovation has time-varying spectral structure, hence, it is suitable for describing the empirical financial time series data. Due to its nonstationarity, the least squares estimators of these models do not satisfy asymptotic normality. In this paper, the limiting distributions of the least squares estimators of unit root, near unit root, and general integrated processes with LSP innovation are derived.

The paper titled "Statistical portfolio estimation under the utility function depending on exogenous variables" by K. Hamada et al. develops the portfolio estimation under the situation. To estimate the optimal portfolio, a function of sample moments of the return process and sample cumulant between the return processes and exogenous variables is introduced. Then, its asymptotic distribution is derived, and the influence of exogenous variable on the return process is illuminated.

The paper titled "Statistical estimation for CAPM with long-memory dependence" by T. Amano et al. investigates the Capital Asset Pricing Model (CAPM) with time dimension. In view of time series analysis, authors describe the model of CAPM such as a regression model that market portfolio and the error process are long-memory process and correlated each other. They give a sufficient condition for the return of assets in the CAPM to be short memory. In this setting, they propose a two-stage least squares estimator for the regression coefficient and derive the asymptotic distribution. Some numerical studies are given.

This issue develops a modern, high-level statistical optimal estimation theory for portfolio coefficients, assuming that the financial returns are "dependent and non-Gaussian," which opens up a new horizon in the field of portfolio estimation.

Masanobu Taniguchi Cathy W. S. Chen Junichi Hirukawa Hiroshi Shiraishi Kenichiro Tamaki David Veredas 


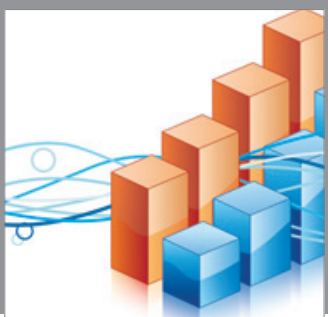

Advances in

Operations Research

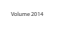

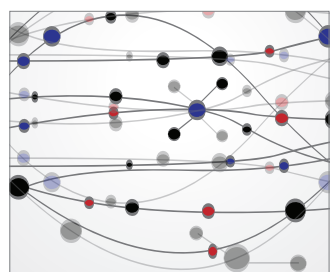

\section{The Scientific} World Journal
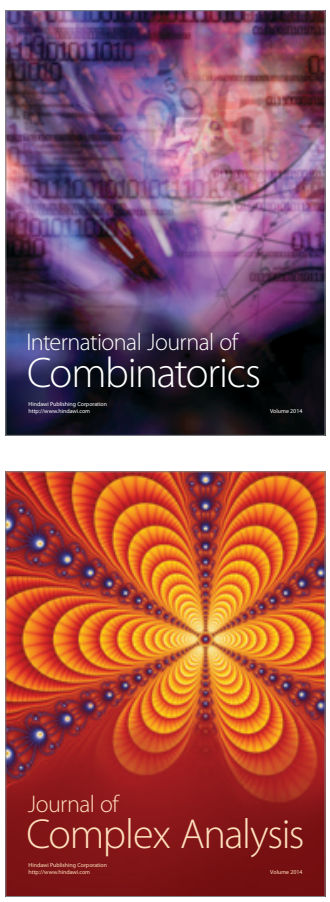

International Journal of

Mathematics and

Mathematical

Sciences
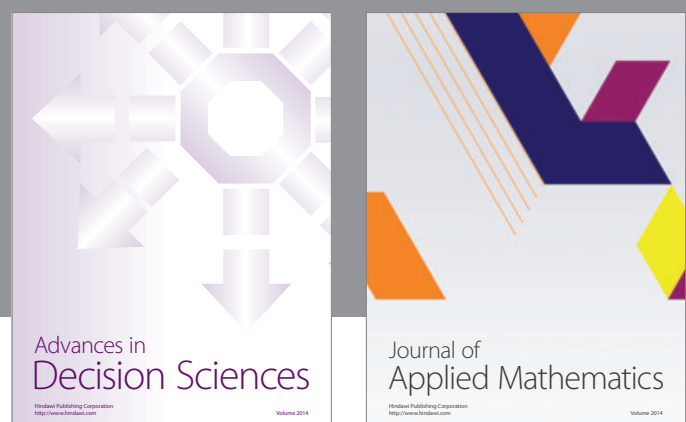

Journal of

Applied Mathematics
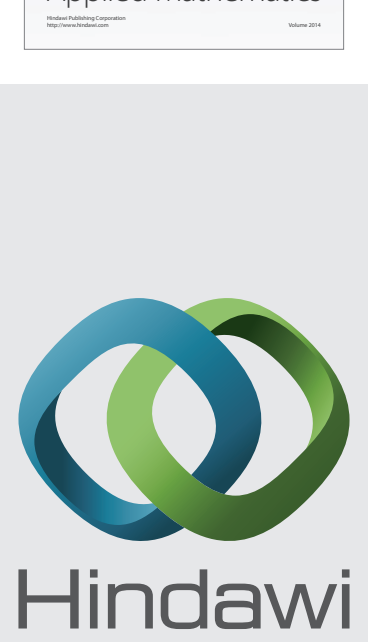

Submit your manuscripts at http://www.hindawi.com
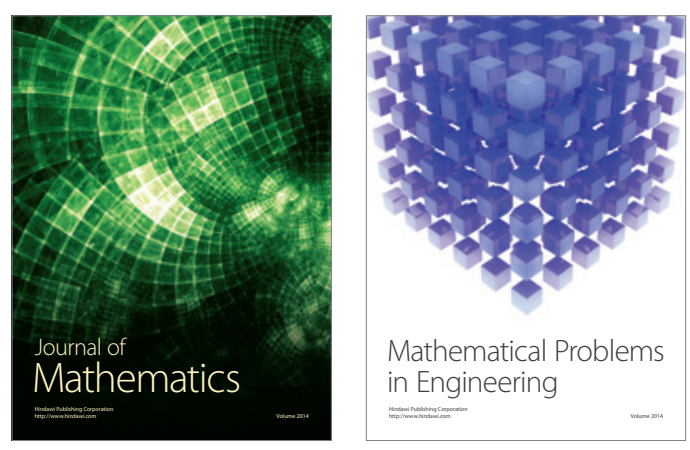

Mathematical Problems in Engineering
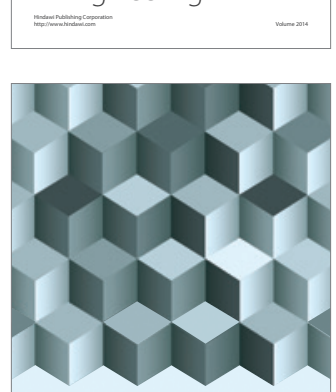

Journal of

Function Spaces
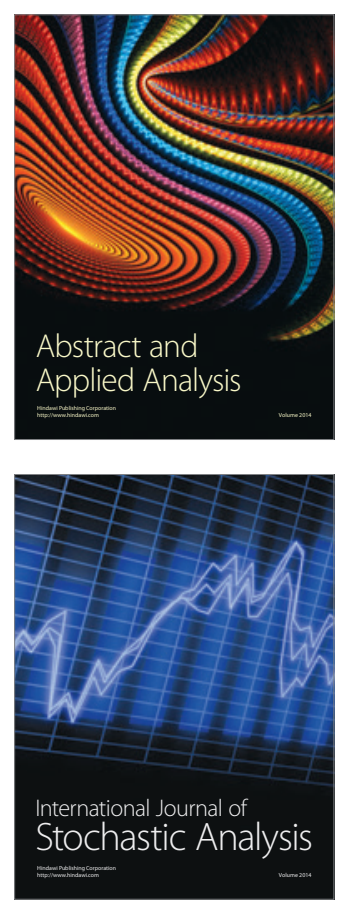

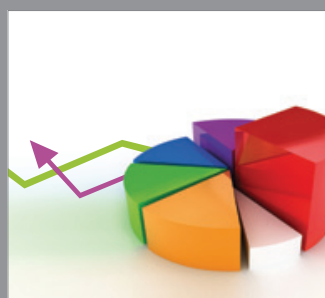

ournal of

Probability and Statistics

Promensencen
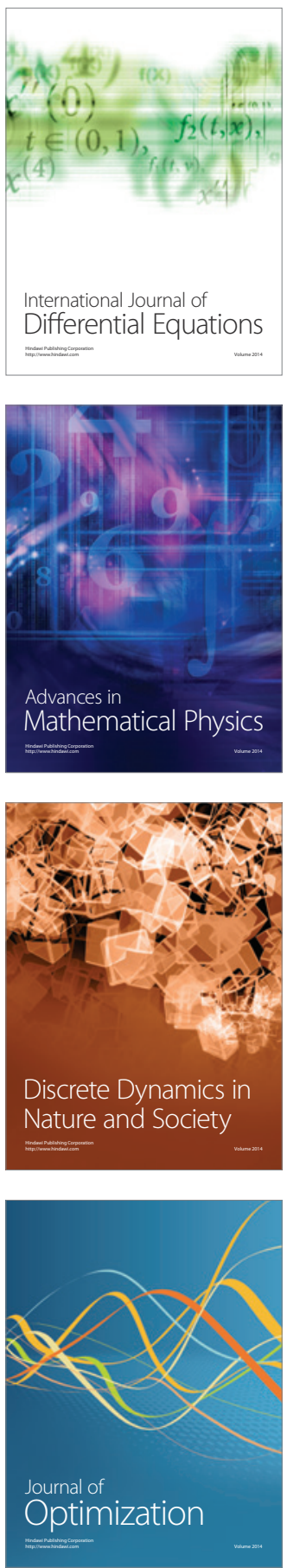